Article

\title{
Perpetual American Cancellable Standard Options in Models with Last Passage Times
}

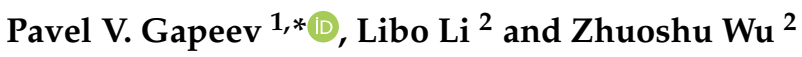 \\ 1 Department of Mathematics, London School of Economics, Houghton Street, London WC2A 2AE, UK \\ 2 School of Mathematics and Statistics, University of New South Wales, Sydney, NSW 2052, Australia; \\ libo.li@unsw.edu.au (L.L.); zhuoshu.wu@student.unsw.edu.au (Z.W.) \\ * Correspondence: p.v.gapeev@lse.ac.uk
}

check for updates

Citation: Gapeev, P.V.; Li, L.; Wu, Z. Perpetual American Cancellable Standard Options in Models with Last Passage Times. Algorithms 2021, 14, 3. https://dx.doi.org/10.3390/a14010003

Received: 26 November 2020 Accepted: 19 December 2020 Published: 24 December 2020

Publisher's Note: MDPI stays neutral with regard to jurisdictional claims in published maps and institutional affiliations.

Copyright: (C) 2020 by the authors. Licensee MDPI, Basel, Switzerland. This article is an open access article distributed under the terms and conditions of the Creative Commons Attribution (CC BY) license (https: / creativecommons.org/ licenses/by/4.0/).

\begin{abstract}
We derive explicit solutions to the perpetual American cancellable standard put and call options in an extension of the Black-Merton-Scholes model. It is assumed that the contracts are cancelled at the last hitting times for the underlying asset price process of some constant upper or lower levels which are not stopping times with respect to the observable filtration. We show that the optimal exercise times are the first times at which the asset price reaches some lower or upper constant levels. The proof is based on the reduction of the original optimal stopping problems to the associated free-boundary problems and the solution of the latter problems by means of the smooth-fit conditions.
\end{abstract}

Keywords: perpetual American options; optimal stopping problem; Brownian motion; first passage time; last hitting time; free-boundary problem; instantaneous stopping and smooth fit; a change-ofvariable formula with local time on surfaces

\section{Introduction}

The main aim of this paper is to compute closed-form expressions for the value functions of the discounted optimal stopping problems:

$$
\bar{V}(x)=\sup _{\tau} E\left[e^{-r \tau}\left(L-X_{\tau}\right) I(\tau<\theta)\right] \quad \text { and } \quad \bar{U}(x)=\sup _{\zeta} E\left[e^{-r \zeta}\left(X_{\zeta}-K\right) I(\zeta<\eta)\right]
$$

with some $L, K>0$ fixed, where $I(\cdot)$ denotes the indicator function.

Here, for precise formulation of the problems, we consider a probability space $(\Omega, \mathcal{F}, P)$ with a standard Brownian motion $B=\left(B_{t}\right)_{t \geq 0}$. Here, the process $X=\left(X_{t}\right)_{t \geq 0}$ is given by:

$$
X_{t}=x \exp \left(\left(r-\delta-\sigma^{2} / 2\right) t+\sigma B_{t}\right)
$$

which solves the stochastic differential equation:

$$
d X_{t}=(r-\delta) X_{t} d t+\sigma X_{t} d B_{t} \quad\left(X_{0}=x\right)
$$

where $x>0$ is fixed, and $r>0, \delta>0$, and $\sigma>0$ are some given constants. Assume that the process $X$ describes the price of risky assets on a financial market, where $r$ is the riskless interest rate, $\delta$ is the dividend rate paid to the asset holders, and $\sigma$ is the volatility rate. We also introduce the random times $\theta$ and $\eta$ by:

$$
\theta=\sup \left\{t \geq 0 \mid X_{t} \geq h\right\} \text { and } \eta=\sup \left\{t \geq 0 \mid X_{t} \leq g\right\}
$$

for some $h>L>0$ and $0<g<K$ fixed, which are not stopping times with respect to the natural filtration $\left(\mathcal{F}_{t}\right)_{t>0}$ of the process $X$, but they are honest times in the sense of Nikeghbali and Yor [1]. Assume that the process $X$ describes the price of a risky asset in 
a financial market, where $r$ is the riskless interest rate, $\delta$ is the dividend rate paid to the asset holders, and $\sigma$ is the volatility rate. Suppose that the suprema in (1) are taken over all stopping times $\tau$ and $\zeta$ with respect to the filtration $\left(\mathcal{F}_{t}\right)_{t \geq 0}$, and the expectations there are taken with respect to the risk-neutral probability measure $P$. In this view, the values $\bar{V}$ and $\bar{U}$ in (1) are the no-arbitrage prices of the perpetual American cancellable options in the Black-Merton-Scholes model (see, e.g., [2] ([Chapter VII, Section 3g])). Note that other perpetual American cancellable options with another game-type payoff structure were recently studied by Emmerling [3] (see also references therein). Some extensive overviews of the perpetual American options in diffusion models of financial markets and other related results in the area are provided in Shiryaev [2] ([Chapter VIII; Section 2a]), Peskir and Shiryaev [4] ([Chapter VII; Section 25]), and Detemple [5] among others.

The model studied here differs from models studied in existing works such as Szimayer [6], Gapeev and Al Motairi [7], Glover and Hulley [8], Dumitrescu et al. [9], and Grigorova et al. [10], as neither the immersion hypothesis nor the density hypothesis is satisfied by the random times (or default times) $\theta$ and $\eta$, and the default intensity process simply does not exists in our setting (see, e.g., Bielecki and Rutkowski [11]). We see clearly in (6) and (7) that, in the case of zero recovery, this leads to a modified discounting factors, which are no longer functions of the sum of the interest rate and the default intensity rate. In addition, the diversion from the immersion hypothesis leads to the appearance of an adjusted dividend rate. Finally, if we were to study the finite horizon problem from a point of view of the backward stochastic differential equations (or BSDEs) as in [9,10], then it could be shown that the dynamics of the no-arbitrage (pre-default) price will no longer satisfy a linear reflected BSDE but rather a linear reflected generalised BSDE where the generalised driver is related to the local time of the underlying asset at $h$ or $g$.

We further study the problems of (1) as the associated optimal stopping problems of (20) and (21) for the one-dimensional continuous Markov underlying risky asset price process $X$. Note that the integrals in the reward functionals of the optimal stopping problems in (20) and (21) contain local times of the process $X$ at the points $h$ and $g$ which represents an interesting feature for the general theory of optimal stopping problems for continuous Markov processes.

The rest of the paper is organised as follows. In Section 2, we embed the original problems of (1) into the optimal stopping problems of (20) and (21) for the one-dimensional continuous Markov process $X$ defined in (2). It is shown that the optimal exercise times $\tau^{*}$ and $\zeta^{*}$ are the first times at which the process $X$ reaches some lower or upper constant levels $a^{*}$ or $b^{*}$. In Section 3, we derive explicit expressions for the associated value functions $V^{*}(x)$ and $U^{*}(x)$ as solutions to the equivalent free-boundary problems and apply the smooth-fit conditions to characterise the optimal stopping boundaries $a^{*}$ and $b^{*}$. In Section 4 , by using the change-of-variable formula with local time on surfaces from Peskir [12], we verify that the solutions of the free-boundary problems provide the solutions of the original optimal stopping problems. The main results of the paper are stated in Theorem 1.

\section{Preliminaries}

In this section, we introduce the setting and notation of the two-dimensional optimal stopping problems which are related to the pricing of perpetual American cancellable standard put-and-call options and formulate the equivalent free-boundary problems.

\subsection{The Optimal Stopping Problems}

In order to compute the expectations in (1), let us now introduce the conditional survival processes $Z=\left(Z_{t}\right)_{t \geq 0}$ and $Y=\left(Y_{t}\right)_{t \geq 0}$ defined by $Z_{t}=P\left(\theta>t \mid \mathcal{F}_{t}\right)$ and $Y_{t}=P\left(\eta>t \mid \mathcal{F}_{t}\right)$, for all $t \geq 0$, respectively. Note that the processes $Z$ and $Y$ are called the Azéma supermartingales of the random times $\theta$ and $\eta$ (see, e.g., [13] ([Section 1.2.1])). By using the fact that the running maximum of a Brownian motion with the drift coefficient $r-\delta-\sigma^{2} / 2<0$ has an exponential distribution with the mean $1 /\left(\sigma^{2}+2(\delta-r)\right)$, while the running minimum of a Brownian motion with the drift coefficient $r-\delta-\sigma^{2} / 2>0$ 
has an exponential distribution with the mean $1 /\left(2(r-\delta)-\sigma^{2}\right)$ (see, e.g., [14] ([Chapter II, Exercise 3.12])), we have:

$$
Z_{t}=\left\{\begin{array}{ll}
\left(h / X_{t}\right)^{\alpha} \wedge 1, & \text { if } \alpha<0 \\
1, & \text { if } \alpha \geq 0
\end{array} \text { and } \quad Y_{t}= \begin{cases}\left(g / X_{t}\right)^{\alpha} \wedge 1, & \text { if } \alpha>0 \\
1, & \text { if } \alpha \leq 0\end{cases}\right.
$$

where we set $\alpha=2(r-\delta) / \sigma^{2}-1$. The representations in (5) can also be obtained by applying Doob's maximal equality (see, e.g., [13] ([Lemma 0.1]) or [14] ([Chapter II, Exercise 3.12])) to the process $X^{-\alpha}=\left(X_{t}^{-\alpha}\right)_{t \geq 0}$, which is a strictly positive continuous local martingale, and thus, a supermartingale converging to zero at infinity. Then, it follows from a direct application of the tower property for conditional expectations that the first terms in the right-hand sides of the expressions in (1) have the form:

$$
E\left[e^{-r \tau}\left(L-X_{\tau}\right) I(\tau<\theta)\right]=E\left[e^{-r \tau}\left(L-X_{\tau}\right)\left(\left(h / X_{\tau}\right)^{\alpha} \wedge 1\right)\right]
$$

when $\alpha<0$, and

$$
E\left[e^{-r \zeta}\left(X_{\zeta}-K\right) I(\zeta<\eta)\right]=E\left[e^{-r \zeta}\left(X_{\zeta}-K\right)\left(\left(g / X_{\zeta}\right)^{\alpha} \wedge 1\right)\right]
$$

when $\alpha>0$, for any stopping times $\tau$ and $\zeta$ of the process $X$. It is seen from the structure of the rewards in (1) with (6) and (7) that it is not optimal to exercise the perpetual American cancellable put or call options, when $X_{t}>L$ or $X_{t}<K$ holds, for any $t \geq 0$, respectively.

By applying the change-of-variable formula from [12] ([Theorem 3.1]) (see also [4] ([Chapter II, Section 3.5]) for a summary of the related results and further references) to the process $e^{-r t} G_{1}\left(X_{t}\right)$ with $G_{1}(x)=(L-x)\left((h / x)^{\alpha} \wedge 1\right)$, for $x>0$, we obtain the representation:

$$
\begin{aligned}
& e^{-r t} G_{1}\left(X_{t}\right)=G_{1}(x) \\
& +\int_{0}^{t} e^{-r u}\left(G_{1}^{\prime}\left(X_{u}\right)(r-\delta) X_{u}+G_{1}^{\prime \prime}\left(X_{u}\right) \frac{\sigma^{2} X_{u}^{2}}{2}-r G_{1}\left(X_{u}\right)\right) I\left(X_{u} \neq h\right) d u \\
& +\int_{0}^{t} e^{-r u}\left(G_{1}^{\prime}(h+)-G_{1}^{\prime}(h-)\right) I\left(X_{u}=h\right) d \ell_{u}^{h}(X)+N_{t}^{1}
\end{aligned}
$$

when $\alpha<0$, for each $x>0$ and all $t \geq 0$. Here, the process $N^{1}=\left(N_{t}^{1}\right)_{t \geq 0}$ given by:

$$
N_{t}^{1}=\int_{0}^{t} e^{-r u} G_{1}^{\prime}\left(X_{u}\right)\left(h / X_{u}\right)^{\alpha} I\left(X_{u} \neq h\right) \sigma d B_{u}
$$

is a continuous uniformly integrable martingale under the probability measure $P$, when $\alpha<0$, while the process $\ell^{h}(X)=\left(\ell_{t}^{h}(X)\right)_{t \geq 0}$ defined as the limit in probability by:

$$
\ell_{t}^{h}(X)=P-\lim _{\varepsilon \downarrow 0} \frac{1}{2 \varepsilon} \int_{0}^{t} I\left(h-\varepsilon<X_{u}<h+\varepsilon\right) \sigma^{2} X_{u}^{2} d u
$$

is the local time of the process $X$ at the point $h$. Then, by means of Doob's optional sampling theorem (see, e.g., [15] ([Chapter III, Theorem 3.6]) or [14] ([Chapter II, Theorem 3.2])), we get:

$$
\begin{aligned}
& E\left[e^{-r \tau} G_{1}\left(X_{\tau}\right)\right]=G_{1}(x) \\
& +E\left[\int_{0}^{\tau} e^{-r u}\left(G_{1}^{\prime}\left(X_{u}\right)(r-\delta) X_{u}+G_{1}^{\prime \prime}\left(X_{u}\right) \frac{\sigma^{2} X_{u}^{2}}{2}-r G_{1}\left(X_{u}\right)\right) I\left(X_{u} \neq h\right) d u\right. \\
& \left.\quad+\int_{0}^{\tau} e^{-r u}\left(G_{1}^{\prime}(h+)-G_{1}^{\prime}(h-)\right) I\left(X_{u}=h\right) d \ell_{u}^{h}(X)\right]
\end{aligned}
$$

when $\alpha<0$, for any stopping time $\tau$ with respect to $\left(\mathcal{F}_{t}\right)_{t \geq 0}$. Hence, getting the expressions in (11) together with the ones in (6) above, we may conclude that the value $\bar{V}(x)$ from 
(1) is given by:

$$
\begin{aligned}
\bar{V}(x)=G_{1}(x)+\sup _{\tau} E[ & \int_{0}^{\tau} e^{-r u} H_{1}\left(X_{u}\right) I\left(X_{u} \neq h\right) d u \\
& \left.+\int_{0}^{\tau} e^{-r u}\left(G_{1}^{\prime}(h+)-G_{1}^{\prime}(h-)\right) I\left(X_{u}=h\right) d \ell_{u}^{h}(X)\right]
\end{aligned}
$$

when $\alpha<0$, for each $x>0$, where the supremum is taken over all stopping times $\tau$ of the process $X$. Here, we put:

$$
\begin{aligned}
H_{1}(x) & =G_{1}^{\prime}(x)(r-\delta) x+G_{1}^{\prime \prime}(x) \frac{\sigma^{2} x^{2}}{2}-r G_{1}(x) \\
& =\left(\delta^{\prime} x-r L\right)(h / x)^{\alpha} I(x<h)+(\delta x-r L) I(x \geq h)
\end{aligned}
$$

for all $x>0$, where we set $\delta^{\prime}=\delta+\alpha \sigma^{2} \equiv 2 r-\delta-\sigma^{2}$, that can be considered as a cancellation adjusted dividend rate.

Moreover, by applying the change-of-variable formula from [12] ([Theorem 3.1]) to the process $e^{-r t} G_{2}\left(X_{t}\right)$ with $G_{2}(x)=(x-K)\left((g / x)^{\alpha} \wedge 1\right)$, for $x>0$, we obtain the representation:

$$
\begin{aligned}
& e^{-r t} G_{2}\left(X_{t}\right)=G_{2}(x) \\
& +\int_{0}^{t} e^{-r u}\left(G_{2}^{\prime}\left(X_{u}\right)(r-\delta) X_{u}+G_{2}^{\prime \prime}\left(X_{u}\right) \frac{\sigma^{2} X_{u}^{2}}{2}-r G_{2}\left(X_{u}\right)\right) I\left(X_{u} \neq g\right) d u \\
& +\int_{0}^{t} e^{-r u}\left(G_{2}^{\prime}(g+)-G_{2}^{\prime}(g-)\right) I\left(X_{u}=g\right) d \ell_{u}^{g}(X)+N_{t}^{2}
\end{aligned}
$$

when $\alpha<0$, for each $x>0$ and all $t \geq 0$, where we have $\delta^{\prime}=\delta+\alpha \sigma^{2} \equiv 2 r-\delta-\sigma^{2}$. Here, the process $N^{2}=\left(N_{t}^{2}\right)_{t \geq 0}$ given by:

$$
N_{t}^{2}=\int_{0}^{t} e^{-r u} G_{2}^{\prime}\left(X_{u}\right)\left(g / X_{u}\right)^{\alpha} I\left(X_{u} \neq g\right) \sigma d B_{u}
$$

is a continuous uniformly integrable martingale under the probability measure $P$, when $\alpha>0$, while the process $\ell^{g}(X)=\left(\ell_{t}^{g}(X)\right)_{t \geq 0}$ defined as the limit in probability by:

$$
\ell_{t}^{g}(X)=P-\lim _{\varepsilon \downarrow 0} \frac{1}{2 \varepsilon} \int_{0}^{t} I\left(g-\varepsilon<X_{u}<g+\varepsilon\right) \sigma^{2} X_{u}^{2} d u
$$

is the local time of the process $X$ at the point $g$. Then, by means of Doob's optional sampling theorem,

$$
\begin{aligned}
& E\left[e^{-r \zeta} G_{2}\left(X_{\zeta}\right)\right]=G_{2}(x)+E\left[\int_{0}^{\zeta} e^{-r u}\left(G_{2}^{\prime}\left(X_{u}\right)(r-\delta) X_{u}+G_{2}^{\prime \prime}\left(X_{u}\right) \frac{\sigma^{2} X_{u}^{2}}{2}-r G_{2}\left(X_{u}\right)\right) I\left(X_{u} \neq g\right) d u\right. \\
& \left.\quad+\int_{0}^{\zeta} e^{-r u}\left(G_{2}^{\prime}(g+)-G_{2}^{\prime}(g-)\right) I\left(X_{u}=g\right) d \ell_{u}^{g}(X)\right]
\end{aligned}
$$

when $\alpha>0$, for any stopping time $\zeta$ with respect to $\left(\mathcal{F}_{t}\right)_{t \geq 0}$. Hence, getting the expressions in (17) together with the ones in (7) above, we may conclude that the value $\bar{U}(x)$ from (1) is given by:

$$
\begin{aligned}
\bar{U}(x)=G_{2}(x)+\sup _{\zeta} E[ & \int_{0}^{\zeta} e^{-r u} H_{2}\left(X_{u}\right) I\left(X_{u} \neq g\right) d u \\
& \left.+\int_{0}^{\zeta} e^{-r u}\left(G_{2}^{\prime}(g+)-G_{2}^{\prime}(g-)\right) I\left(X_{u}=g\right) d \ell_{u}^{g}(X)\right]
\end{aligned}
$$


when $\alpha>0$, for each $x>0$, where the supremum is taken over all stopping times $\zeta$ of the process $X$. Here, we put:

$$
\begin{aligned}
H_{2}(x) & =G_{2}^{\prime}(x)(r-\delta) x+G_{2}^{\prime \prime}(x) \frac{\sigma^{2} x^{2}}{2}-r G_{2}(x) \\
& =\left(r K-\delta^{\prime} x\right)(g / x)^{\alpha} I(x>g)+(r K-\delta x) I(x \leq g)
\end{aligned}
$$

for all $x>0$, where we recall that $\delta^{\prime}=\delta+\alpha \sigma^{2} \equiv 2 r-\delta-\sigma^{2}$. Note that, since the time spent by the process $X$ at the constant boundaries $h$ and $g$ is of the Lebesgue measure zero (see, e.g., [16] ([Chapter II, Section 1])), the indicators in the expressions of (12) and (18) can be set equal to one.

Therefore, we see that the problems in (12) can be naturally embedded into the optimal stopping problems for the (time-homogeneous strong) Markov process $X$ with the value functions:

$$
V^{*}(x)=\sup _{\tau} E_{x}\left[\int_{0}^{\tau} e^{-r u} H_{1}\left(X_{u}\right) d u+\int_{0}^{\tau} e^{-r u}\left(G_{1}^{\prime}(h+)-G_{1}^{\prime}(h-)\right) I\left(X_{u}=h\right) d \ell_{u}^{h}(X)\right]
$$

when $\alpha<0$, and

$$
U^{*}(x)=\sup _{\zeta} E_{x}\left[\int_{0}^{\zeta} e^{-r u} H_{2}\left(X_{u}\right) d u+\int_{0}^{\zeta} e^{-r u}\left(G_{2}^{\prime}(g+)-G_{2}^{\prime}(g-)\right) I\left(X_{u}=g\right) d \ell_{u}^{g}(X)\right]
$$

when $\alpha>0$, respectively. Here, $E_{x}$ denotes the expectation with respect to the probability measures $P_{x}$ under which the one-dimensional Markov process $X$ defined in (2) starts at $x>0$. We further obtain solutions to the optimal stopping problems in (20) and (21) and verify below that the value functions $V^{*}(x)$ and $U^{*}(x)$ are the solutions of the problems in (12) and (18), and thus, give the solutions of the original problems in (1).

\subsection{The Structure of Optimal Exercise Times}

Let us now determine the structure of the optimal stopping times at which the holders should exercise the contracts. We first note that, it follows from the structure of the first integrands in (20) and (21) that it is not optimal to exercise the perpetual American cancellable put option when $H_{1}\left(X_{t}\right) \geq 0$, while it is not optimal to exercise the corresponding call option when $H_{2}\left(X_{t}\right) \geq 0$, for any $t \geq 0$, respectively. In this respect, if we assume that $\delta^{\prime} \equiv 2 r-\delta-\sigma^{2} \leq 0$ holds, that obviously implies that $\alpha \equiv 2(r-\delta) / \sigma^{2}-1<0$ holds, then we see from the expression in (20) that the equality $\tau^{*}=0$ should hold for the optimal stopping time, so that one should exercise the perpetual American cancellable put option instantly. In this view, for simplicity of presentation, we further assume that $\delta^{\prime}>0$ holds, as well as note that the fact that $\alpha \equiv 2(r-\delta) / \sigma^{2}-1>0$ holds obviously implies that $\delta^{\prime} \equiv 2 r-\delta-\sigma^{2}>0$ holds. In this case, the inequality $\left(\delta^{\prime} x-r L\right)(h / x)^{\alpha} \geq 0$ is satisfied if and only if $x \geq \bar{a}$ holds with $\bar{a}=r L / \delta^{\prime}$. Furthermore, the inequality $\left(r K-\delta^{\prime} x\right)(g / x)^{\alpha} \geq 0$ is satisfied if and only if $x \leq \underline{b}$ holds with $\underline{b}=r K / \delta^{\prime}$.

We further search for the optimal stopping times in the problems of (20) and (21) with the structure:

$$
\tau^{*}=\inf \left\{t \geq 0 \mid X_{t} \leq a^{*}\right\} \quad \text { and } \quad \zeta^{*}=\inf \left\{t \geq 0 \mid X_{t} \geq b^{*}\right\}
$$

where the constant boundaries $0<a^{*}<L<h$ and $b^{*}>K>g>0$ are to be determined, when $\alpha<0$ or $\alpha>0$, respectively (see Figures 1 and 2 below for computer drawings of the value functions and the optimal exercise boundaries). 


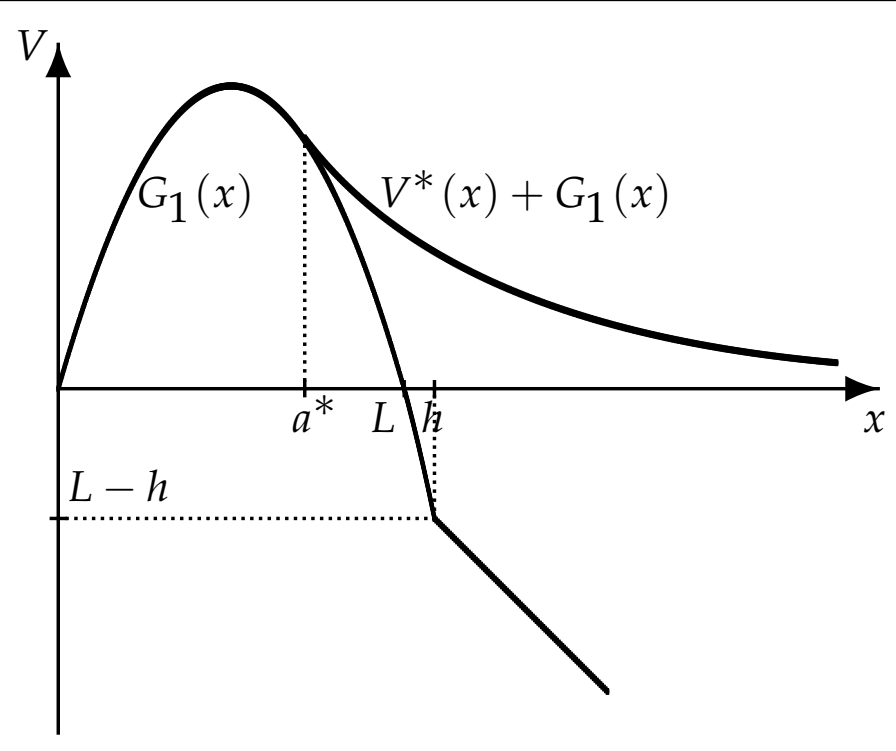

Figure 1. A computer drawing of the value function $V^{*}(x)+G_{1}(x)$.

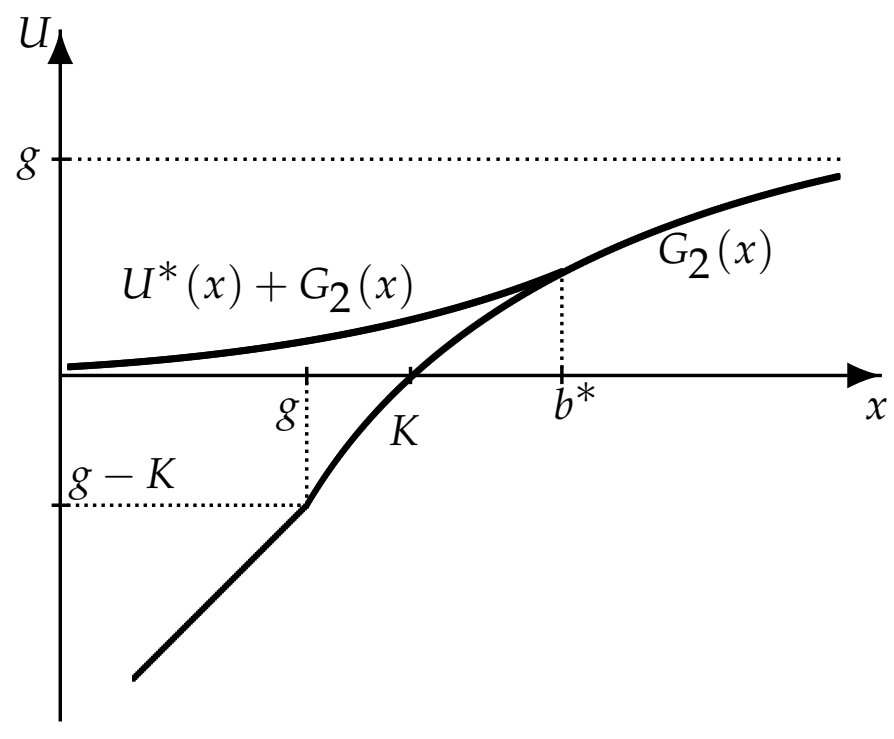

Figure 2. A computer drawing of the value function $U^{*}(x)+G_{2}(x)$.

\subsection{The Free-Boundary Problems}

By means of standard arguments based on the application of Itô's formula (see, e.g., [15] ([Theorem 4.4]) or [14] ([Chapter II, Theorem 3.2])), it is shown that the infinitesimal operator $\mathbb{L}$ of the process $X$ from (3) acts on a function $F(x)$ from the class $C^{2}$ according to the rule:

$$
(\mathbb{L} F)(x)=(r-\delta) x F^{\prime}(x)+\frac{\sigma^{2} x^{2}}{2} F^{\prime \prime}(x)
$$

for all $x>0$ (see, e.g., [17] ([Chapter V, Section 5.1])). In order to find analytic expressions for the unknown value functions $V^{*}(x)$ and $U^{*}(x)$ from (20) and (21) and the unknown 
boundaries $a^{*}$ and $b^{*}$ from (22), we apply the results of general theory for solving optimal stopping problems for Markov processes presented in [4] ([Chapter IV, Section 8]) among others. More precisely, for the original optimal stopping problems in (20) and (21), we formulate the associated free-boundary problems (see, e.g., [4] ([Chapter IV, Section 8])) and then verify in Theorem 4.1 below that the appropriate candidate solutions of the latter problems coincide with the solutions of the original problems. In other words, we reduce the optimal stopping problems of (20) and (21) to the following equivalent free-boundary problems:

$$
\begin{aligned}
(\mathbb{L} V-r V)(x)=-H_{1}(x) \text { for } x>a, & (\mathbb{L} U-r U)(x)=-H_{2}(x) \text { for } x<b \\
\left.V(x)\right|_{x=a+}=0, & \left.U(x)\right|_{x=b-}=0 \\
\left.V^{\prime}(x)\right|_{x=a+}=0, & \left.U^{\prime}(x)\right|_{x=b-}=0 \\
V(x)=0 \text { for } x<a, & U(x)=0 \text { for } x>b \\
V(x)>0 \text { for } x>a, & U(x)>0 \text { for } x<b \\
(\mathbb{L} V-r V)(x)<-H_{1}(x) \text { for } x<a, & (\mathbb{L} U-r U)(x)<-H_{2}(x) \text { for } x>b
\end{aligned}
$$

for some $0<a<L<h$ and $b>K>g>0$, where the functions $H_{1}(x)$ and $H_{2}(x)$ have the form of (13) and (19), respectively. Observe that the superharmonic characterisation of the value function (see, e.g., [4] ([Chapter IV, Section 9])) implies that $V^{*}(x)$ and $U^{*}(x)$ are the smallest functions satisfying (24),(25) and (27),(28) with the boundaries $a^{*}$ and $b^{*}$, respectively. Note that the inequalities in (29) follow directly from the arguments of Section 2.2 above.

\section{Solutions to the Free-Boundary Problems}

In this section, we obtain solutions to the free-boundary problems in (24)-(29) and derive first-order nonlinear ordinary differential equations for the candidate optimal stopping boundaries.

\subsection{The Candidate Value Functions}

It is shown that the second-order ordinary differential equations in (24) have the general solutions:

$$
V(x)=C_{1} x^{\gamma_{1}}+C_{2} x^{\gamma_{2}}+(x-L)(h / x)^{\alpha} I(x<h)+(x-L) I(x \geq h)
$$

when $\alpha<0$, and

$$
U(x)=D_{1} x^{\gamma_{1}}+D_{2} x^{\gamma_{2}}+(K-x)(g / x)^{\alpha} I(x>g)+(K-x) I(x \leq g)
$$

when $\alpha>0$, for all $x>0$, respectively. Here, $C_{i}$ and $D_{i}$, for $i=1,2$, are some arbitrary constants, and $\gamma_{i}$, for $i=1,2$, are given by:

$$
\gamma_{i}=\frac{1}{2}-\frac{r-\delta}{\sigma^{2}}-(-1)^{i} \sqrt{\left(\frac{1}{2}-\frac{r-\delta}{\sigma^{2}}\right)^{2}+\frac{2 r}{\sigma^{2}}}
$$

so that $\gamma_{2}<0<1<\gamma_{1}$ holds. Observe that $C_{1}=0$ and $D_{2}=0$ should hold for the candidate value functions in (30) and (31), since otherwise $V(x) \rightarrow \pm \infty$ as $x \uparrow \infty$ and $U(x) \rightarrow \pm \infty$ as $x \downarrow 0$, that must be excluded, by virtue of the fact that the values $V^{*}(x)$ and $U^{*}(x)$ in (20) and (21) are finite. Then, by applying the conditions of (25) and (26) to the functions in (30) and (31), we obtain the equalities:

$$
\begin{aligned}
& C_{2} a^{\gamma_{2}}+(a-L)(h / a)^{\alpha}=0 \\
& \gamma_{2} C_{2} a^{\gamma_{2}}+((1-\alpha) a+L \alpha)(h / a)^{\alpha}=0
\end{aligned}
$$


for $a<h$, and

$$
\begin{aligned}
& D_{1} b^{\gamma_{1}}+(K-b)(g / b)^{\alpha}=0 \\
& \gamma_{1} D_{1} b^{\gamma_{1}}-((1-\alpha) b+K \alpha)(g / b)^{\alpha}=0
\end{aligned}
$$

for $b>g$, respectively. Hence, by solving the systems of equations in (33), (34) and (35), (36), we obtain that the candidate value functions admit the representations:

$$
V(x ; a)=(L-a)(x / a)^{\gamma_{2}}(h / a)^{\alpha}+(x-L)(h / x)^{\alpha} I(x<h)+(x-L) I(x \geq h)
$$

for $x>a$ with $a<L<h$, and

$$
U(x ; b)=(b-K)(x / b)^{\gamma_{1}}(g / b)^{\alpha}+(K-x)(g / x)^{\alpha} I(x>g)+(K-x) I(x \leq g)
$$

for $x<b$ with $b>K>g$, respectively.

Moreover, by means of straightforward computations, it can be deduced from the expressions in (37) and (38) that the first- and second-order derivatives $V^{\prime}(x ; a)$ and $V^{\prime \prime}(x ; a)$ of the function $V(x ; a)$ take the form:

$$
\begin{aligned}
V^{\prime}(x ; a)= & (L-a)\left(\gamma_{2} / a\right)(x / a)^{\gamma_{2}-1}(h / a)^{\alpha}+(1-\alpha)(h / x)^{\alpha} I(x<h) \\
& +L(\alpha / x)(h / x)^{\alpha} I(x<h)+I(x \geq h)
\end{aligned}
$$

and

$$
\begin{aligned}
V^{\prime \prime}(x ; a)= & (L-a)\left(\gamma_{2}\left(\gamma_{2}-1\right) / a^{2}\right)(x / a)^{\gamma_{2}-2}(h / a)^{\alpha}-((1-\alpha) \alpha / x)(h / x)^{\alpha} I(x<h) \\
& -L\left(\alpha(\alpha+1) / x^{2}\right)(h / x)^{\alpha} I(x<h)
\end{aligned}
$$

on the interval $x>a$, while the first- and second-order derivatives $U^{\prime}(x ; b)$ and $U^{\prime \prime}(x ; b)$ of the function $U(x ; b)$ take the form:

$$
\begin{aligned}
U^{\prime}(x ; b)= & (b-K)\left(\gamma_{1} / b\right)(x / b)^{\gamma_{1}-1}(g / b)^{\alpha}-(1-\alpha)(g / x)^{\alpha} I(x>g) \\
& -K(\alpha / x)(g / x)^{\alpha} I(x>g)-I(x \leq g)
\end{aligned}
$$

and

$$
\begin{aligned}
U^{\prime \prime}(x ; b)= & (b-K)\left(\gamma_{1}\left(\gamma_{1}-1\right) / b^{2}\right)(x / b)^{\gamma_{1}-2}(g / b)^{\alpha}+((1-\alpha) \alpha / x)(g / x)^{\alpha} I(x>g) \\
& +K\left(\alpha(\alpha+1) / x^{2}\right)(g / x)^{\alpha} I(x>g)
\end{aligned}
$$

respectively.

\subsection{The Candidate Stopping Boundaries}

By solving the systems of equations in (33), (34) and (35), (36), we obtain that the candidate exercise boundaries have the form:

$$
a^{*}=\frac{\gamma_{2}+\alpha}{\gamma_{2}+\alpha-1} L \quad \text { and } \quad b^{*}=\frac{\gamma_{1}+\alpha}{\gamma_{1}+\alpha-1} K
$$

when $\alpha<0$ or $\alpha>0$, under the assumption $\delta^{\prime} \equiv 2 r-\delta-\sigma^{2}>0$, respectively. Moreover, it is shown by means of straightforward computations that the inequalities:

$$
a^{*}<\frac{r L}{\delta^{\prime}} \text { and } b^{*}>\frac{r K}{\delta^{\prime}}
$$

hold with $\delta^{\prime} \equiv 2 r-\delta-\sigma^{2}>0$, so that the appropriate inequalities (29) are satisfied. 


\section{Main Results and Proofs}

In this section, based on the previous computations, we formulate and prove our main results.

Theorem 1. Let the processes $X$ be given by (2), with some $r>0, \delta>0$, and $\sigma>0$, and the inequality $\delta^{\prime} \equiv 2 r-\delta-\sigma^{2}>0$ be satisfied. Suppose that the random times $\theta$ and $\eta$ are defined by (4), which are honest times but not stopping times with respect to the natural filtration $\left(\mathcal{F}_{t}\right)_{t>0}$ of the process X. Then, the value functions of the perpetual American cancellable standard put and call options from (20) and (21) admit the expressions:

$$
V^{*}(x)= \begin{cases}V\left(x ; a^{*}\right), & \text { if } x>a^{*} \\ 0, & \text { if } 0<x \leq a^{*}\end{cases}
$$

whenever $\alpha \equiv 2(r-\delta) / \sigma^{2}-1<0$, and

$$
U^{*}(x)= \begin{cases}U\left(x ; b^{*}\right), & \text { if } 0<x<b^{*} \\ 0, & \text { if } x \geq b^{*}\end{cases}
$$

whenever $\alpha>0$. Here, the function $V(x ; a)$ admits the representation of (37) and the optimal exercise boundary $a^{*}$ is given by (43), whenever $\alpha<0$, while $U(x ; b)$ admits the representation of (38) and the optimal exercise boundary $b^{*}$ is given by (43), whenever $\alpha>0$.

Since both parts of the assertion stated above are proved using similar arguments, we only give a proof for the case of the one-dimensional optimal stopping problem of (21) related to the perpetual American cancellable standard call options.

Proof. In order to verify the assertion stated above, it remains for us to show that the function defined in (46) coincides with the value function in (21) and that the stopping time $\zeta^{*}$ in (22) is optimal with the boundary $b^{*}$ specified above. Let us denote by $U(x)$ the right-hand side of the expression in (46). Then, it is shown by means of straightforward calculations from the previous section that the function $U(x)$ solves the right-hand system of (24)-(29). Observe that the function $U(x)$ is $C^{2}$ on the closures $\{x>0 \mid 0<x \leq g\}$ and $\left\{x>0 \mid g \leq x \leq b^{*}\right\}$, while it is equal to zero on the closed set $\left\{x>0 \mid x \geq b^{*}\right\}$. Hence, by applying the change-of-variable formula from [12] ([Theorem 3.1]) to the process $e^{-r t} U\left(X_{t}\right)$, we obtain the expression:

$$
\begin{aligned}
e^{-r t} U\left(X_{t}\right)= & U(x)+\int_{0}^{t} e^{-r u}(\mathbb{L} U-r U)\left(X_{u}\right) I\left(X_{u} \neq g, X_{u} \neq b\right) d u+M_{t} \\
& +\int_{0}^{t} e^{-r u}\left(U^{\prime}(g+)-U^{\prime}(g-)\right) I\left(X_{u}=g\right) d \ell_{u}^{g}(X)
\end{aligned}
$$

for all $t \geq 0$. Here, the process $M=\left(M_{t}\right)_{t \geq 0}$ defined by:

$$
M_{t}=\int_{0}^{t} e^{-r u} U^{\prime}\left(X_{u}\right) I\left(X_{u} \neq g\right) \sigma X_{u} d B_{u}
$$

is a continuous local martingale with respect to the probability measure $P_{x}$. Note that, since the time spent by the process $X$ at the constant boundary $b$ is of the Lebesgue measure zero (see, e.g., [16] ([Chapter II, Section 1])), the indicators in the first line of the expression of (47) as well as in the expression of (48) can be set equal to one.

It follows from straightforward calculations and the arguments of the previous section that the function $U(x)$ satisfies the second-order ordinary differential equation in (24), which together with the right-hand conditions of (25)-(27) as well as the fact that the righthand inequality in (29) holds imply that the inequality $(\mathbb{L} U-r U)(x) \leq-H_{2}(x)$ is satisfied with $H_{2}(x)$ given by (19), for all $x>0$ such that $x \neq b$. Moreover, we observe directly from the expressions in (38), (41) and (42) that the function $U(x)$ is convex and decreases 
to zero, because its first-order derivative $U^{\prime}(x)$ is negative and increases to zero, while its second-order derivative $U^{\prime \prime}(x)$ is positive, on the interval $0<x<b$, under $\alpha>0$. Thus, we may conclude that the inequality in (28) holds, which together with the conditions of ( 25)-(27) imply that the inequality $U(x) \geq 0$ is satisfied, for all $x>0$.

Let $\left(\kappa_{n}\right)_{n \in \mathbb{N}}$ be the localising sequence of stopping times for the process $M$ from (48) such that $\kappa_{n}=\inf \left\{t \geq 0|| M_{t} \mid \geq n\right\}$, for each $n \in \mathbb{N}$. We also observe from the explicit expressions in (38) and (41) that the equality $U^{\prime}(g+)-U^{\prime}(g-)=-G_{2}^{\prime}(g+)+G_{2}^{\prime}(g-)$ holds. It therefore follows from the expression in (47) that the inequalities:

$$
\begin{aligned}
& \int_{0}^{\zeta \wedge \kappa_{n}} e^{-r u} H_{2}\left(X_{u}\right) d u+\int_{0}^{\zeta \wedge \kappa_{n}} e^{-r u}\left(G_{2}^{\prime}(g+)-G_{2}^{\prime}(g-)\right) I\left(X_{u}=g\right) d \ell_{u}^{g}(X) \\
& \leq e^{-r\left(\zeta \wedge \kappa_{n}\right)} U\left(X_{\zeta \wedge \kappa_{n}}\right)+\int_{0}^{\zeta \wedge \kappa_{n}} e^{-r u} H_{2}\left(X_{u}\right) d u \\
& \quad+\int_{0}^{\zeta \wedge \kappa_{n}} e^{-r u}\left(G_{2}^{\prime}(g+)-G_{2}^{\prime}(g-)\right) I\left(X_{u}=g\right) d \ell_{u}^{g}(X) \\
& \leq U(x)+M_{\zeta \wedge \kappa_{n}}
\end{aligned}
$$

are satisfied, for any stopping time $\zeta$ of the process $X$ and each $n \in \mathbb{N}$ fixed. Then, taking the expectation with respect to $P_{x}$ in (49), by means of Doob's optional sampling theorem, we obtain

$$
\begin{aligned}
& E_{x}\left[\int_{0}^{\zeta \wedge \kappa_{n}} e^{-r u} H_{2}\left(X_{u}\right) d u+\int_{0}^{\zeta \wedge \kappa_{n}} e^{-r u}\left(G_{2}^{\prime}(g+)-G_{2}^{\prime}(g-)\right) I\left(X_{u}=g\right) d \ell_{u}^{g}(X)\right] \\
& \leq U(x)+E_{x}\left[M_{\zeta \wedge \kappa_{n}}\right]=U(x)
\end{aligned}
$$

for all $x>0$ and each $n \in \mathbb{N}$. Hence by letting $n$ go to infinity and using Fatou's lemma, we obtain from the expressions in (50) that the inequalities:

$$
E_{x}\left[\int_{0}^{\zeta} e^{-r u} H_{2}\left(X_{u}\right) d u+\int_{0}^{\zeta} e^{-r u}\left(G_{2}^{\prime}(g+)-G_{2}^{\prime}(g-)\right) I\left(X_{u}=g\right) d \ell_{u}^{g}(X)\right] \leq U(x)
$$

hold, for any stopping time $\zeta$ and all $x>0$. In order to prove the fact that the boundary $b^{*}$ is optimal, we consider the sequence of stopping times $\kappa_{n}, n \in \mathbb{N}$, defined as in the righthand part of (22). Then, by virtue of the fact that the function $U(x)$ from the right-hand side of the expression in (46) associated with the boundary $b$ satisfies the conditions of (24) and (25), and taking into account the structure of $\zeta^{*}$ in (22), it follows from the expression which is equivalent to the one in (47) that the equalities:

$$
\begin{aligned}
& \int_{0}^{\zeta^{*} \wedge \kappa_{n}} e^{-r u} H_{2}\left(X_{u}\right) d u+\int_{0}^{\zeta^{*} \wedge \kappa_{n}} e^{-r u}\left(G_{2}^{\prime}(g+)-G_{2}^{\prime}(g-)\right) I\left(X_{u}=g\right) d \ell_{u}^{g}(X) \\
& =e^{-r\left(\zeta^{*} \wedge \kappa_{n}\right)} U\left(X_{\zeta^{*} \wedge \kappa_{n}}\right)+\int_{0}^{\zeta^{*} \wedge \kappa_{n}} e^{-r u} H_{2}\left(X_{u}\right) d u \\
& \quad+\int_{0}^{\zeta^{*} \wedge \kappa_{n}} e^{-r u}\left(G_{2}^{\prime}(g+)-G_{2}^{\prime}(g-)\right) I\left(X_{u}=g\right) d \ell_{u}^{g}(X) \\
& =U(x)+M_{\zeta^{*} \wedge \kappa_{n}}
\end{aligned}
$$

hold, for all $x>0$ and each $n \in \mathbb{N}$. Observe that, by virtue of the arguments from [2] ([Chapter VIII, Section 2a]), the property:

$$
E_{x}\left[\sup _{t \geq 0}\left(\int_{0}^{\zeta^{*} \wedge t} e^{-r u} H_{2}\left(X_{u}\right) d u+\int_{0}^{\tau^{*} \wedge t} e^{-r u}\left(G_{2}^{\prime}(g+)-G_{2}^{\prime}(g-)\right) I\left(X_{u}=g\right) d \ell_{u}^{g}(X)\right)\right]<\infty
$$

holds, for all $x>0$. Hence, letting $n$ go to infinity and using the condition of (25), we can apply the Lebesgue dominated convergence theorem in the expression of (52) to obtain 
the equality:

$$
E_{x}\left[\int_{0}^{\zeta^{*}} e^{-r u} H_{2}\left(X_{u}\right) d u+\int_{0}^{\zeta^{*}} e^{-r u}\left(G_{2}^{\prime}(g+)-G_{2}^{\prime}(g-)\right) I\left(X_{u}=g\right) d \ell_{u}^{g}(X)\right]=U(x)
$$

for all $x>0$, which together with the inequalities in (51) directly implies the desired assertion.

Author Contributions: Conceptualisation, L.L.; methodology, Z.W.; writing—original draft, P.V.G.; writing - review and editing, L.L., Z.W. All authors have read and agreed to the published version of the manuscript.

Funding: This research received no external funding.

Conflicts of Interest: The authors declare no conflict of interest.

\section{References}

1. Nikeghbali, A.; Yor, M. Doob's maximal identity, multiplicative decomposition and enlargement of filtrations. Ill. J. Math. 2006, 50, 791-814. [CrossRef]

2. Shiryaev, A.N. Essentials of Stochastic Finance; World Scientific: Singapore, 1999.

3. Emmerling, T.J. Perpetual cancellable American call option. Math. Financ. 2012, 22, 645-666. [CrossRef]

4. Peskir, G.; Shiryaev, A.N. Optimal Stopping and Free-Boundary Problems; Birkhäuser: Basel, Switzerland, 2006.

5. Detemple, J. American-Style Derivatives: Valuation and Computation; Chapman and Hall/CRC: Boca Raton, FL, USA, 2006.

6. Szimayer, A. Valuation of American options in the presence of event risk. Financ. Stoch. 2005, 9, 89-107. [CrossRef]

7. Gapeev, P.V.; Al Motairi, H. Perpetual American defaultable options in models with random dividends and partial information. Risks 2018, 6, 127. [CrossRef]

8. Glover, K.; Hulley, H. Short Selling with Margin Risk and Recall Risk. Working Paper. arXiv 2019, arXiv:1903.11804.

9. Dumitrescu, R.; Quenez, M.C.; Sulem, A. American options in an imperfect complete market with default. ESAIM Proc. Surv. 2018, 64, 93-110. [CrossRef]

10. Grigorova, M.; Quenez, M.C.; Sulem, A. American Options in a Non-Linear Incomplete Market Model with Default. Working Paper. 2019. Available online: https:/ /hal.archives-ouvertes.fr/hal-02025835/document (accessed on 24 December 2020)

11. Bielecki, T.R.; Rutkowski, M. Credit Risk: Modeling, Valuation and Hedging, 2nd ed.; Springer: Berlin, Germany, 2004.

12. Peskir, G. A change-of-variable formula with local time on surfaces. In Séminaire de Probabilité XL; Lecture Notes in Mathematics 1899; Springer: Berlin, Germany, 2007; pp. 69-96.

13. Mansuy, R.; Yor, M. Random Times and Enlargements of Filtration in a Brownian Setting; Lecture Notes in Mathematics 1873; Springer: Berlin, Germany, 2006.

14. Revuz, D.; Yor, M. Continuous Martingales and Brownian Motion; Springer: Berlin, Germany, 1999.

15. Liptser, R.S.; Shiryaev, A.N. Statistics of Random Processes I, 2nd ed.; First Edition 1977; Springer: Berlin, Germany, 2001.

16. Borodin, A.N.; Salminen, P. Handbook of Brownian Motion, 2nd ed.; Birkhäuser: Basel, Switzerland, 2002.

17. Karatzas, I.; Shreve, S.E. Brownian Motion and Stochastic Calculus, 2nd ed.; Springer: New York, NY, USA, 1991. 